Zaur M. Gamishidze

Batumi Shota Rustaveli State University, zauri5erambler.ru, Batumi-Georgia

DOI $\quad$ http://dx.doi.org/10.12739/NWSA.2017.12.4.3A0081

\title{
THE EFFECT OF SMALL SUBSTITUTIONS OF Gd ON THE MAGNETIC PROPERTIES OF SYSTEM $Y\left(\operatorname{Co}_{1-x} A \ell_{x}\right)_{2}$
}

\begin{abstract}
Compound $\mathrm{YCo}_{2}$ is a zonal metamagnet. It was found that partial substitution of cobalt for aluminum in $\mathrm{YCo}_{2}$ leads to a decrease in the matamagnetic transition field $H_{m}$ and the emergence of weak zonal ferromagnetism in the system $Y\left(\operatorname{Co}_{1-x} A \ell_{x}\right)_{2}$ at $x \geq 0.12$. This can significantly modify the character of the effect of rare-earth impurities on the $d$-subsystem. To exclude the effect of the crystalline field, gadolinium was used as a magnetizing element. Hence the magnetic properties of the system $Y_{1-t} G d_{t}\left(C o_{1-x} A \ell_{x}\right)_{2}$ were studied in this work. We confined ourselves to the aluminum concentration range $(0 \leq x \leq 0.105)$ in the limits of which initial compounds $Y\left(C o_{1-x} A \ell_{x}\right)_{2}$ are paramagnets and undergo a metamagnetic transition in the field. It is shown that, with an increase in the concentration of $G d$ (with constant concentration of $A \ell$ ), the transition from paramagnetism to ferromagnetism takes place. When the content of $A \ell$ increases, the area of ferromagnetism expands, and the paramagnetic state area shrinks. The behavior of the compounds under study in the field depends on the ratio between the degrees of magnetization of cobalt $M_{C o}$ and gadolinium $M_{G d}$ subsystems. If $M_{C o}$ in a weak field is greater than $M_{G d}$, gadolinium impurities shift the metamagnetic transition field towards weak fields, as the molecular field acting on cobalt from the gadolinium subsystem $H_{m}$ is summed with the external magnetic field $H$. In case $M_{C o}$ in a weak field is smaller than $M_{G d}, H$ and $H_{m}$ are directed antiparallel to each other.

Keywords: Metamagnetizm, Magnetization, Susceptibility, Density of States, Exchange Interaction
\end{abstract}

\section{INTRODUCTION}

Recently, when studying the specific features of magnetism of metals and alloys, the phenomena associated with the magnetic instability of the subsystem of zonal d-electrons have aroused considerable interest. One of characteristic manifestations of such instability is zonal metamagnetism induced by the field of the firstorder phase transition from the paramagnetic to ferromagnetic state. This phenomenon is associated with a special form of energy dependence of the density of states in the vicinity of the Fermi level, which facilitates an increase in $N(\varepsilon)$ with applied magnetic field at $H<H_{m}$ $I\left(\varepsilon_{F}\right)<1$ and at $H>H_{m} \quad I\left(\varepsilon_{F}\right)>1$, where $I\left(\varepsilon_{F}\right)$ is the coefficient of exchange interaction [1]. When the coefficient of exchange interaction is positive, the exchange interaction strives to orient the spins of electrons (and hence the magnetic moments) parallel to each other. As a result, the exchange interaction enhances paramagnetism. As the calculations showed, magnetic susceptibility of the exchange- 
intensified paramagnet $\chi_{e x}$ increases in comparison with the susceptibility of free electron gas $\chi_{p}[1]$ :

$$
\chi_{e x}=\frac{\chi_{p}}{1-\lambda \chi_{p}}
$$

where $\lambda=I / \mu_{B}^{2}$ is the molecular field coefficient for the $f-d$ exchange interaction ( $\lambda$ is negative for compounds with heavy rare earths). The metamagnetic transition occurs in the paramagnetic system in the critical field $H_{m}$ when the stoner criterion begins to be fulfilled, $I N\left(\varepsilon_{F}\right) \geq 1$. Thus, in the system of collectivized electrons, ferromagnetism is a threshold phenomenon, it occurs only when the Stoner criterion is fulfilled, i.e. at relatively big values of the exchange interaction parameter $I$ and high density of states at the Fermi level $N\left(\varepsilon_{F}\right)$ [2 and 3]. Currently, there are known few objects in which zonal metamagnetism is experimentally observed. That is why the phenomenon of zonal magnetism is poorly understood.

\section{RESEARCH SIGNIFICANCE}

It was found that partial substitution of cobalt for aluminum in $\mathrm{YCo}_{2}$ leads to a decrease in the metamagnetic transition field $H_{m}$ and emergence of weak zonal metamagnetism in the system $Y\left(\operatorname{Co}_{1-x} A \ell_{x}\right)_{2}$ at $x \geq 0.12$ [4 and 5]. Such evolution of the properties of these compounds is explained in the scope of the zonal magnetism model by an increase in the density of states at the Fermi level with substitution of cobalt for aluminum. Thus, in the system $Y\left(\operatorname{Co}_{1-x} A \ell_{x}\right)_{2}$, magnetic stability of a $d$-subsystem increases with the increasing aluminum concentration. This can significantly modify the character of the effect of rare-earth impurities on the $d$-subsystem. However, currently the effect of small rare-earth impurities on the properties of the $d$-subsystem is poorly understood. Hence in this work we studied the magnetic properties of the system $Y_{1-t} G d_{t}\left(C o_{1-x} A \ell_{x}\right)_{2}$ with the aim to clarify the role of the $f-d$ exchange interaction in the magnetic behavior of the zonal system $Y\left(C_{1-x} A \ell_{x}\right)_{2}$ at low concentrations of the localized impurity. We limited ourselves to the concentration range of aluminum $(0 \leq x \leq 0.105)$ where the initial compounds $Y\left(\mathrm{Co}_{1-x} A \ell_{x}\right)_{2}$ are paramagnets and undergo the metamagnetic transition in the field. Thus, we synthesized polycrystalline systems $Y_{1-t} G d_{t}\left(\operatorname{Co}_{1-x} A \ell_{x}\right)_{2}$.

\section{EXPERIMENTAL METHOD-PROCESS}

Polycrystaline samples of intermetalic compounds $Y_{1-t} G d_{t}\left(\mathrm{Co}_{1-x} A \ell_{x}\right)_{2}$, where $0 \leq t \leq 0.20$ and $x=0,0.05,0.07,0.085,0.105$, were smelted in an induction furnace in the atmosphere of spectrally pure argon in a water-cooled copper crucible under quasilevitation. The produced ingots were homogenized at $850^{\circ} \mathrm{C}$ for a week. The magnetization was measured in static magnetic fields up to 60 kOe and pulse magnetic fields up to $300 \mathrm{kOe}$ over the range from $4.2 \mathrm{~K}$ to 300K. The magnetic susceptibility was measured in weak alternating fields over the same temperature range. The basic properties of studied compounds $Y_{1-t} G d_{t}\left(\mathrm{Co}_{1-x} A \ell_{x}\right)_{2}$ can be theoretically discussed in the framework of the average field model. Let us consider the zonal $d-$ subsystem of these compounds in the scope of the Wohlfahrt model of weak zonal ferromagnetism [4 and 6]. Then, taking into account the $f-d$ exchange interaction, we can write equation of state for the zonal subsystem in the form

$$
\alpha \vec{M}_{d}+\beta \vec{M}_{d}^{3}+\gamma \vec{M}_{d}^{5}=\vec{H}_{e f f}^{d}
$$

where $\vec{M}_{d}$ is the magnetization of the $d$-subsystem, $\vec{H}_{e f}^{d}$ is the effective field acting this subsystem:

$$
\vec{H}_{e f f}^{d}=\vec{H}+\lambda_{f d} t \vec{M}_{f}
$$


Where $\vec{H}_{m o l}^{d}=\lambda_{f d} t \vec{M}_{f}$ is the molecular field acting the $d$-subsystem, $\lambda_{f d}$ is the coefficient of the molecular field of $f-d$ exchange interaction (for the compounds of heavy rare-earth elements, $\lambda_{f d}$ is negative), $M_{f}$ is the magnetization of the $f$-subsystem (calculated for a single $f$ ion). If the magnetization of the $d$-subsystem is more than that of the $f$-subsystem $\left(M_{d}>t M_{f}\right)$, the magnetic moment of the $d$-subsystem is oriented parallel to the external field, while the moment of the $f$ subsystem is antiparallel to it, and the $f-d$ exchange interaction leads to a decrease in the metamagnetic transition field

$$
H_{M}(t)=H_{M}(0)-\left(-\lambda_{f d} t M_{f}\right)
$$

$\left(t \lambda_{f d}<0\right)$. If the magnetization of the $d$-subsystem is less than that of the $f$-subsystem $\left(M_{d}<t M_{f}\right)$, the magnetic moment of the $d$-subsystem is oriented antiparallel to the external field, while the moment of the $f$-subsystem-along the field. Then, when the external field is applied, the $d$-subsystem is first demagnetized and then established in parallel to the external field, and the $f-d$ exchange field causes an increase in the field of the metamagnetic transition

$$
H_{M}(t)=H_{M}(O)+\left(-\lambda_{f d} t M_{f}\right)
$$

\section{RESULTS AND DISCUSSION}

The measurements, we carried out, showed that low-temperature magnetic properties of the systems $Y_{1-t} G d_{t}\left(C o_{1-x} A \ell_{x}\right)_{2}$ sharply depend on the concentration of aluminum. In Fig. 1 are shown magnetization curves of some compositions of the system $Y_{1-t} G d_{t}\left(\mathrm{Co}_{0.915} A \ell_{0.085}\right)_{2}$ at $4.2 \mathrm{~K}$. It is seen that the initial compound $Y\left(\mathrm{Co}_{0.915} A \ell_{0.085}\right)_{2}$ is a zonal metamagnet (Figure 1, curve 1). The critical field of metamagnetic transition for this compound makes up 225kOe. It is seen that, as $Y$ is substituted for $G d$, the field $H_{m}$ decreases monotonously, and, at concentrations $t \geq$ 0.04, these compounds possess spontaneous magnetization. It happens due to that the exchange field $H_{f-d}$ helps the external field with magnetization. It is important to note that, at the mentioned concentrations of gadolinium in this system, the magnetization of the $d$-subsystem is directed along the field. The transition field in substituted compositions is very simply related to the metamagnetic transition field of pure compounds $\left(\mathrm{Co}_{1-x} A \ell_{x}\right)_{2} H_{M}(t)=H_{M}(O)-\lambda_{f d} t \mu_{f}$, where $t$ is the

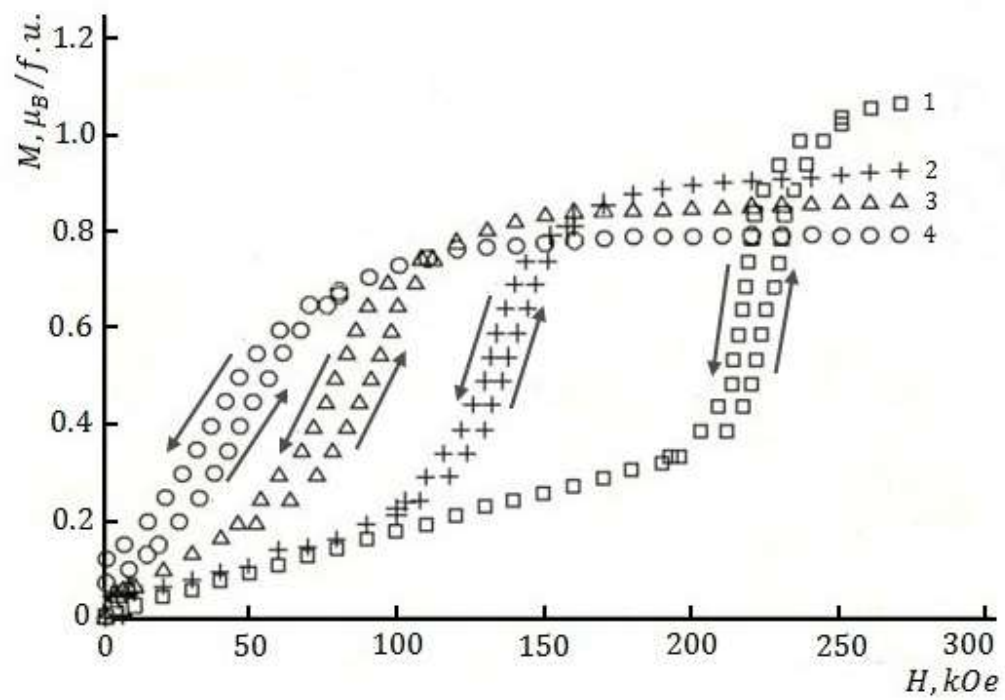

Figure 1. 1Magnetization curves of the compounds $Y_{1-t} G d_{t}\left(\mathrm{Co}_{0.915} A \ell_{0.085}\right)_{2}$ at $4.2 K$ and $t=0.0(1), 0.02(2), 0.03(3) 0.04(4)$ 
concentration of $G d, \mu_{f}$ is the magnetic moment of $G d, \lambda_{f d}$ is the molecular field coefficient. The calculations showed that, already in the fields $\sim 30 k 0 e, \mu_{f}$ can be considered equal to $7 \mu_{B}$ with high accuracy. From this it is easy to determine the molecular field coefficient $\lambda_{f d}$, which turned out to be practically independent of the concentrations of $G d$ and $A \ell$. In Figure 2 is shown the dependence of the metamagnetic transition field on the gadolinium concentration $t$ for the system $Y_{1-t} G d_{t}\left(\mathrm{Co}_{1-x} A \ell_{x}\right)_{2}$. It is seen that, as predicted theoretically [7, 8], metamgnetic transition fields of the systems with high concentration of aluminum $(x=0.07,0.085$ and 0.105$)$, in which the susceptibility of the $d$-subsystem is high, decrease practically linearly with the increasing concentration of gadolinium.

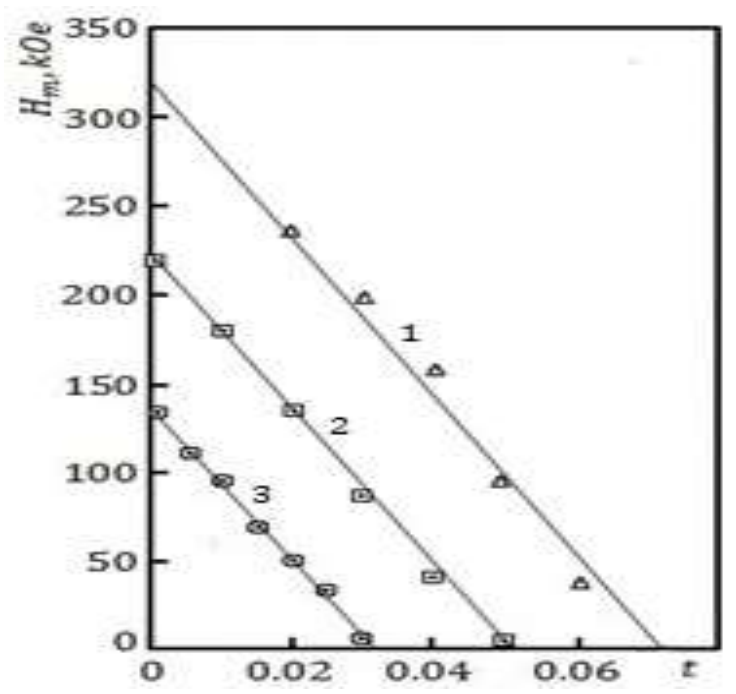

Figure 2. Dependence of the metamagnetic transition field on the gadolinium concentration for the system $Y_{1-t} G d_{t}\left(C_{0.915} A \ell_{0.085}\right)_{2}: x=0.07(1)$, 0.085(2), 0.105(3). The straight line is calculated theoretical, and the symbols are experimental data

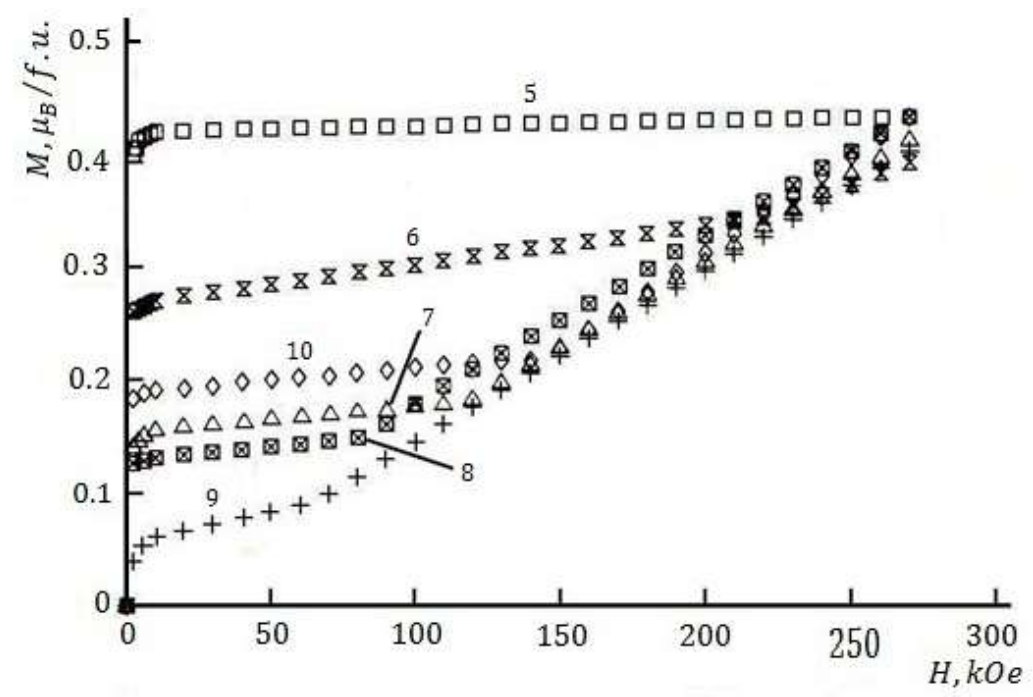

Figure 3. Magnetization curves of the compounds $Y_{1-t} G d_{t}\left(\mathrm{Co}_{0.915} A \ell_{0.085}\right)_{2}$ at $4.2 K$ and $t=0.10(5), t=0.12(6), 0.14(7), 0.15(8), 0.18(9), 0.20(10)$

A completely different pattern of magnetization is observed when $M_{d}<M_{f}$ (Figure 3). In such a case, the external field demagnetizes the $d$-subsystem and does not magnetize it, and there are observed no 
metamagnetic transitions. In the zero field, such compounds are in a strongly ferrimagnetic state. In most cases it corresponds to the concentration of $10 \div 14 \% \mathrm{Gd}$. A specific feature of all studied samples is that saturation magnetization decreases with the decreasing concentration of gadolinium. At magnetization of about 18 at.\%Gd, the degrees of $d$-magnetization of $f$ - and $d$-subsystems become equal and then increase with increasing saturation magnetization. In the vicinity of this concentration, the transitions from a collinear ferrimagnetic phase to a noncollinear phase in the external magnetic field take place. Near the points of magnetic compensation fieldinduced noncollinear magnetic structures can be observed. Based on these data and using a simple formula $H_{c 1}=\lambda_{f d}\left|M_{f}-M_{d}\right|$, the constant $\lambda_{f d}$ can be determined [9 and 10].

\section{CONCLUSION}

The comparison of experimental data with the calculated ones for the $f-d$ magnetic system showed that they are in many ways consistent, at least qualitatively. Firstly, in most cases theoretically predicted transitions from the paramagnetic phase with metamagnetism to a weakly ferromagnetic phase and then to a strongly ferromagnetic phase occur when the concentration of gadolinium increases. Secondly, as was theoretically predicted, metamagnetic transition fields of the systems with high concentrations of aluminum ( $x=0.07,0.085$ and 0.105$)$, in which the susceptibility of the $d$-subsystem is high, decrease practically linearly with the increasing concentrations of gadolinium. Thirdly, there were not detected metamagnetic transitions in the systems with low concentrations of aluminum $(x=0.050 .00)$. This is likely to be connected with low susceptibility of the subsystem, because of which in these compounds the mmagnetic moment of the $f$-subsystem is oriented along the field.

\section{NOTE}

This work is presented at 5-8 September 2017, $2^{\text {nd }}$ International Science Symposium (ISS2017) in Tbilisi-Georgia.

\section{REFERENCES}

1. Levitin, R.Z. and Markosyan, A.S., (1988). Usp. Fiz. Nauk 155, 623 [Sov. Phys. Usp. 31, 730 (1988)].

2. Goto, T., Fukamichi, K., Sakakibara, T., (1989). Komatsu H. Sol. St. Comm. Vol:72, pp:945.

3. Mater, (1991). Vol:3, pp:2515.

4. Bloch, D., Edwards, D.M., Shimisu, M., and Voiron, J.J., (1975). Phys. F. Vol:5, pp:1217.

5. Kirchmaier, K.R. and Poldi, K.A., (1982). Physics and Chemistry of Rare-Earth Elements, K.A. Gschneider and L. Airing, eds., Moscow: Metallurgiya.

6. Duc, N.H., Hien, T.D., Brommer, P.E., and France, J.J., (1998). M.L. Phys. F. Vol:18, pp:275.

7. Aleksandryan, V.V., Lagutin, A.S., Levitin, R.Z., at al., (1985). Zh. Eksp. Teor. Fiz. 89:271. [Sov. Phys. JETP 62, 153.

8. Ballou, R., Barbara, B., Gamishidze, Z.M., Lemaire, R., Levitin, R.Z., Markosyan, A.S., Magn, J., (1993). Magn. Mater. 119, 294.

9. Goto, T., Aruga Katori, H., Koui, K., Levitin, R.Z., Markosyan, A.S., and Gamishidze, Z.M., (1994). Field Induced Transitions of $Y_{1-t} G d_{t}\left(\mathrm{Co}_{1-0.93} A \ell_{0.07}\right)_{2}$ in Ultrahigh Magnetic Fields up tu $100 \mathrm{~T}$, Phys B 201 .

10. Tjiablikov, S.V., (1965). In: Methods of Quantum Theory of Magnetism (Niuka, Moscow, 1965) in Russian. 